\title{
Corela
}

Cognition, représentation, langage

9-2 | 2011

Vol. $9, \mathrm{n}^{\circ} 2$

\section{Will dans les subordonnées en if}

\section{Bénédicte Guillaume}

\section{OpenEdition}

\section{Journals}

Édition électronique

URL : http://journals.openedition.org/corela/2298

DOI : $10.4000 /$ corela.2298

ISSN : 1638-573X

\section{Éditeur}

Cercle linguistique du Centre et de l'Ouest - CerLICO

\section{Référence électronique}

Bénédicte Guillaume, «Will dans les subordonnées en if », Corela [En ligne], 9-2 | 2011, mis en ligne le 30 novembre 2011, consulté le 23 avril 2019. URL : http://journals.openedition.org/corela/2298 ; DOI : $10.4000 /$ corela.2298

Ce document a été généré automatiquement le 23 avril 2019

\section{(c) (i) (2)(2)}

Corela - cognition, représentation, langage est mis à disposition selon les termes de la licence Creative Commons Attribution - Pas d'Utilisation Commerciale - Partage dans les Mêmes Conditions 4.0 International. 


\title{
Will dans les subordonnées en if
}

\author{
Bénédicte Guillaume
}

\section{Introduction ${ }^{1}$}

1 L'objet $\mathrm{du}$ présent article est d'étudier l'emploi du modal will ${ }^{2}$ dans certaines subordonnées introduites par la conjonction if, principalement les subordonnées conditionnelles et également certaines concessives. Le cadre théorique auquel il est fait référence est celui de la Théorie des Opérations Enonciatives (TOE) d'A. Culioli. Précisons également que nous excluons du champ de la présente étude les exemples illustrant uniquement la valeur de « volonté » fréquemment associée à l'emploi radical de will's.

2 Nous avons constitué un corpus d'une centaine d'énoncés authentiques collectés au sein du British National Corpus ${ }^{4}$ (BNC). Notre propos ici est avant tout de rendre compte de l'exploitation de ce corpus, dans la mesure où les auteurs s'étant penchés sur la question notent dans leur ensemble la relative rareté des exemples (par exemple Huddleston \& Pullum 2002 : 191), et plus particulièrement la rareté des exemples authentiques (Palmer 1979 : 118). La taille du BNC, tout comme le logiciel de concordance SARA ${ }^{5}$ qui permet de l'interroger, sont de ce fait des atouts garantissant une assez bonne diversité des cas de figure, même si nous ne prétendons pas pour autant à l'exhaustivité.

Nous souhaitons montrer que l'emploi de will dans les exemples qui nous intéressent permet avant tout à l'énonciateur d'effectuer une modalisation ${ }^{6}$ de son énoncé, qui ne traduit pas tant un jugement subjectif de sa part que la reformulation d'implications présentes au sein d'un contexte foncièrement non-neutre. Ces implications sont parfois liées à la problématique du marquage des références temporelles, sans pour autant que cela soit toujours le cas, loin s'en faut. On doit également souligner le rôle prépondérant du complémentaire linguistique de la relation prédicative contenue dans la subordonnée, par rapport auquel la principale peut se trouver repérée. Du reste, les composantes de la tentative de typologie que nous proposons ici ne sont pas hermétiques les unes par rapport aux autres, dans la mesure où plusieurs facteurs peuvent se combiner afin d'expliquer le recours à will dans la subordonnée. 


\section{Opposition au niveau du marquage des références temporelles}

4 En ce qui concerne les conditionnelles ${ }^{7}$ dans un premier temps, la présence de will dans la subordonnée pour faire référence à un événement futur dans un contexte présent est inacceptable la plupart du temps ${ }^{8}$ (tout comme le serait le futur simple en français ${ }^{9}$ ) :

(1) If the weather is nice tomorrow, we will go swimming.

vs. *If the weather will be nice tomorrow, we will go swimming.

$\rightarrow$ S'il fait beau demain, nous irons nager.

vs. *''il fera beau demain, nous irons nager.

On peut opposer l'exemple (1), qui est inventé, aux exemples authentiques suivants, extraits du $\mathrm{BNC}^{10}$ :

(2) The preserved GMP can sometimes fall short of what you would have had from SERPS, and in these cases the state makes up the difference. If you will reach the age of 60 before $1998^{11}$, you can work out very roughly what your SERPS will be in a series of steps.

$\rightarrow$ Le minimum retraite garanti par l'employeur peut parfois être en-deçà de ce que vous auriez obtenu dans le cadre du régime public de retraite lié au revenu, et dans de tels cas de figure l'Etat prend en charge la différence. Si vous devez atteindre l'âge de 60 ans avant 1998, vous pouvez calculer approximativement ce à quoi vous donne droit le régime public en suivant les étapes.

(3) Up till now, anyone who was away on holiday when an election was held lost the right to vote, and some people who were away because of their work lost their vote too. Now the law has changed. As long as there is a good reason why you will not be able to vote in person on election day you can apply to vote by post or proxy. This leaflet tells you how: if you will be away on holiday (in the UK or abroad); if your work will take you away from home; if you have moved house since October 10 last; if you are ill; if you are a student living away from home.

$\rightarrow$ Jusqu'à présent, toute personne partie en vacance lorsqu'avaient lieu des élections perdait son droit de vote, de même que certaines des personnes absentes pour raisons professionnelles. Désormais, la loi a changé. A partir du moment où vous avez une bonne raison de ne pas pouvoir voter en personne le jour des élections, vous pouvez demander à voter par correspondance ou par procuration. Cette brochure vous explique comment faire: si vous partez en vacances (au Royaume-Uni ou à l'étranger); si vous vous absentez pour raisons professionnelles; si vous avez déménagé depuis le 10 octobre dernier; si vous êtes malade; si vous êtes éloigné de votre domicile par vos études.

5 En dépit de l'emploi de la deuxième personne you, qui souvent facilite une telle interprétation, c'est bien une variante de la valeur épistémique du modal que l'on rencontre dans ces subordonnées, et non l'expression d'une volonté. On note du reste dans le présent article que les traductions que nous proposons des exemples concernés font régulièrement intervenir le verbe modalisateur «devoir» en français, dans une acception qui est elle aussi liée à l'évaluation des chances de validation de la relation prédicative contenue dans la subordonnée.

6 Ainsi, (2) et (3) sont proches d'exemples qui ont été recensés et commentés par les linguistes anglophones, notamment R.A. Close (1980), ou encore R. Declerck (1984) ${ }^{12}$, qui décrit pour sa part neuf catégories de subordonnées conditionnelles pouvant contenir le modal will. Quant à Close $(1980: 103 ; 107-9)$, il propose, en s'inspirant de F.R. Palmer (1979) et G. Leech (1971), de faire la distinction entre réalisation future supposée (assumed future actuality) et prévisibilité supposée (assumed predictability) ; dans le premier cas, on postule la réalisation future d'un événement afin d'en tirer les conséquences, et will ne 
peut pas être employé pour faire référence au futur dans la subordonnée. En revanche, dans l'autre cas, on évalue comme probables les chances qu'a l'événement de se réaliser, et là encore cette probabilité entraîne des conséquences ; ce cas de figure est pour sa part compatible avec l'emploi de will dans la subordonnée. Cette hypothèse a par ailleurs l'avantage de faire le lien avec les emplois purement radicaux de will dans une subordonnée en if, qui, toujours d'après Close, décrivent également une forme d'éventualité.

7 R.A. Close identifie les exemples de prévisibilité au moyen de la glose If $X$ is predictable, then the consequence is so-and-so. Huddleston et Pullum (2002: 191) proposent le même genre de distinction que Close, en opposant un événement considéré comme possible, correspondant à l'impossibilité d'employer will dans la subordonnée, et le même événement dans un autre contexte, considéré cette fois-ci comme prévisible, et permettant dès lors l'emploi de will ; la glose donnée pour ce deuxième cas est if it is the case that...

8 Les gloses de ces linguistes, et plus particulièrement celle de Huddleston et Pullum, fonctionnent bien pour les exemples (2) et (3). Il nous semble toutefois que la différence postulée entre possibilité ou prévisibilité de l'événement n'est pas toujours si facile à faire; quant aux gloses proposées, elles sont en réalité suffisamment générales pour pouvoir fonctionner avec un large spectre de subordonnées en if, y compris certaines dans lesquelles l'emploi de will serait malvenu. Il nous paraît donc souhaitable d'affiner les circonstances permettant l'emploi de will.

9 Ainsi, l'exemple (3) déjà cité permet pour sa part une instructive confrontation entre des subordonnées en if au présent simple et des subordonnées en if dans lesquelles will est employé, tandis que sont énumérés les cas de figure pouvant justifier le recours au vote par correspondance ou par procuration (a priori tous mis sur le même plan du point de vue administratif) :

(3') if you will be away on holiday / if your work will take you away from home

vs. if you are ill / if you are a student living away from home

if you have moved house since October 10 last

$(\rightarrow$ ou can apply to vote by post or proxy)

10 En ce qui concerne le marquage des références temporelles, le moment de référence (T1) dans ces exemples correspond à la période pendant laquelle il est possible de faire les démarches pour le vote par correspondance ou par procuration, en général une période de quelques semaines précédant le vote en question. Or, toutes ces subordonnées en if sont repérées par rapport à ce moment $\mathrm{T} 1$, et le recours à will dans certaines d'entre elles permet clairement dans ce cas de marquer un contraste; il s'agit d'opposer les événements ayant déjà cours au moment de référence $\mathrm{T} 1$ (be ill, be a student), qui sont vus comme des états, à des actions plus ponctuelles, quoique prévisibles, telles be away on holiday ou encore be away from home because of your work, qui seront validées à un moment T2 (correspondant au jour des élections) postérieur à T1.

11 Ainsi, le présent employé dans ces subordonnées n'est pas le présent aoristique, c'est-àdire neutre, dénué de toute marque de point de vue, que l'on rencontre pourtant fréquemment dans les subordonnées conditionnelles ${ }^{13}$, mais bel et bien un présent temporel, venant s'opposer à la visée future marquée par will. Il est repéré par rapport à T1, de même que le present perfect de you have moved house traduit pour sa part l'impact présent d'un événement passé. 
12 En ce qui concerne (2), le recours à will peut également se justifier par une opposition marquée entre présent et avenir : if you will reach the age of 60 before 1998 implique que, pour l'instant, c'est-à-dire au moment où la brochure est rédigée et mise à disposition, les personnes concernées n'ont pas encore atteint l'âge de 60 ans.

13 Les exemples (2) et (3) correspondent par ailleurs de manière très exacte aux règles de l'utilisation des temps dans les subordonnées conditionnelles de l'anglais telles que les a décrites B. Comrie (1985: 117-21). D'après ce linguiste en effet, l'emploi du futur (principalement sous la forme will ou shall) est obligatoire lorsque le moment auquel fait référence la subordonnée est postérieur à celui auquel fait référence la principale, à condition qu'il y ait également une relation de cause à effet entre les deux propositions (1985 : 119-20). C'est bien le cas dans les deux exemples qui nous intéressent : en (2), le bénéficiaire n'a pas besoin d'attendre d'avoir atteint un certain âge pour calculer ses droits, mais c'est bien en prévision du jour où il aura cet âge que le calcul s'avère utile ; en (3), il faut faire la demande de vote par correspondance ou par procuration antérieurement à son voyage, mais également en raison de ce dernier.

14 Néanmoins, ce qui retient plus particulièrement notre attention dans ces exemples est l'opposition implicite entre situation présente et situation à venir qui y apparaît, dans la mesure où elle est très proche de celle à laquelle on est confronté lors de l'emploi de will dans certaines subordonnées en when (nous allons revenir sur ce point). En effet, dans de tels exemples, on ne prend pas d'emblée en considération le moment auquel fait référence la subordonnée conditionnelle, qui permettra de déclencher la conséquence énoncée dans la principale. Ce moment est atteint mentalement en partant du moment présent, auquel il s'oppose. Nous rejoignons ici, en la formulant quelque peu différemment, l'opinion de Huddleston et Pullum, qui précisent que l'évaluation des chances que l'événement a de se produire se fait par rapport au moment présent ( $a$ matter of the present predictability of an event »; $2002: 191)^{14}$.

15 Avant d'aborder plus en détail les autres facteurs permettant selon nous l'emploi de will dans certaines subordonnées en if, il est nécessaire d'exposer la justification de l'agrammaticalité de will dans certaines subordonnées telle qu'elle est donnée dans la TOE. Ainsi, lorsque will ne peut absolument pas être employé pour faire référence à un événement futur, comme dans la protase de l'exemple (1), c'est parce que, en termes énonciatifs, la proposition subordonnée fournit le repère de la principale. Ainsi, la relation entre les propositions est de type cause à effet. On peut caractériser ce lien au moyen de la formule if $p$, then $q$, qui correspond à la relation entre la protase et l'apodose telle qu'elle est décrite en grammaire classique ${ }^{15}$. Or, si le modal will n'est guère compatible avec la fonction de repère, c'est parce qu'il modalise l'énoncé en $\mathrm{y}$ introduisant le point de vue de l'énonciateur, ce qui tranche avec le présent simple, dont l'emploi dans de telles subordonnées est pour sa part typiquement aoristique; will introduit donc une dose de subjectivité qui n'en fait pas un bon repère, au sens technique du terme.

16 Afin de pouvoir caractériser le repérage entre propositions de manière plus précise encore, il faut également faire un rappel concernant la construction et la représentation du domaine notionnel dans la TOE, car nous proposons de représenter les relations prédicatives en jeu dans la protase et l'apodose respectivement sous la forme de domaines notionnels. Un domaine notionnel permet en effet de représenter de manière concrète une notion, entité abstraite, qui n'est accessible qu'au travers d'une classe 
d'occurrences phénoménales permettant de dégager des propriétés stables par des opérations d'identification et de différenciation ${ }^{16}$ :

INTERIEUR

propriété $\mathrm{P}$

typique

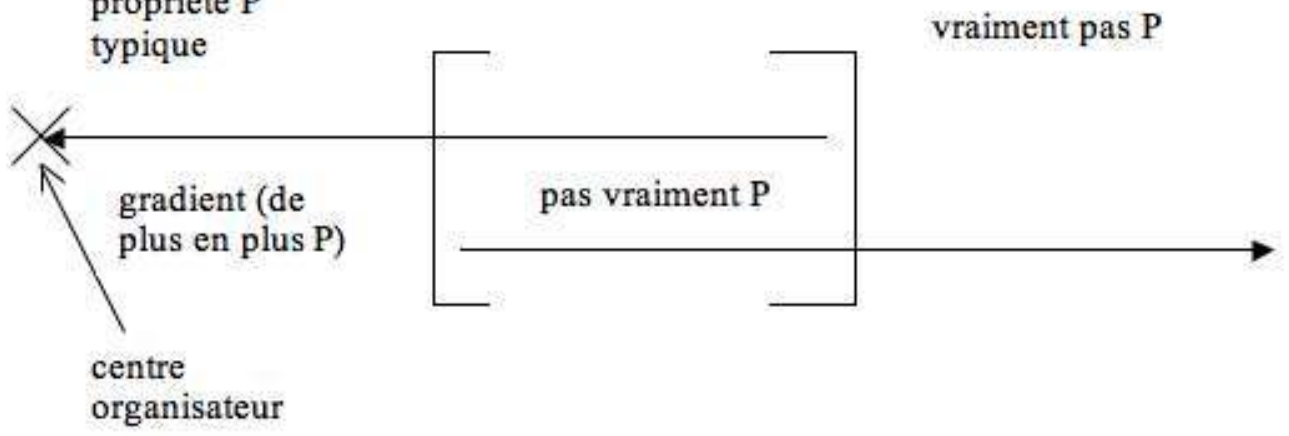

A. Représentation topographique du domaine notionnel dans la TOE (cf. Bouscaren et Chuquet 1987 : 146).

17 Le domaine notionnel se construit autour du centre-organisateur, occurrence typique, possédant la propriété $p$ par excellence. Or, le centre organisateur permet en fait la construction des trois zones distinctes, bien que contiguës, qui composent le domaine notionnel. Tout d'abord, l'Intérieur du domaine notionnel, une zone dans laquelle toutes les occurrences sont identifiées à $p$. On note également la présence d'un gradient, qui correspond à " plus ou moins $p$ ». A la limite entre l'Intérieur et l'Extérieur se trouve la Frontière: c'est une zone où l'on trouve des occurrences présentant la "propriété altérée »; ce n'est ni totalement $p$, ni totalement extérieur. Enfin, l'Extérieur est pour sa part "vide de la propriété constitutive, soit par altérité radicale, soit par inexistence " (Culioli 1990 : 98).

Dans le cas qui nous intéresse, le domaine notionnel permet de représenter la relation prédicative contenue dans la subordonnée ou bien dans la principale. Il s'avère par ailleurs utile d'opposer l'Intérieur strict $p$ à l'Extérieur strict non- $p$ d'une part, et au complémentaire linguistique $p$ ' d'autre part. Ce dernier est constitué de l'Extérieur non-p auquel s'ajoute la Frontière ${ }^{17}$.

19 Ainsi, en reprenant l'exemple (1), l'Intérieur correspond à du beau temps (entrainant la conséquence : «nous irons nager »), tandis que l'Extérieur strict pourrait correspondre à if the weather is bad tomorrow, et qu'un temps incertain, mitigé, correspondrait à la Frontière. Or, sur ces trois cas de figure, seul l'Intérieur est envisagé ; il semble certes sous-entendu que l'on n'ira pas nager en cas de mauvais temps, mais ceci n'est pas totalement explicite. Dans un tel cas de figure, l'emploi de will avec une valeur épistémique dans la subordonnée serait agrammatical, pour les raisons évoquées plus haut: le modal comporterait dès lors une part de subjectivité qui n'est pas compatible avec la fonction de repère. 


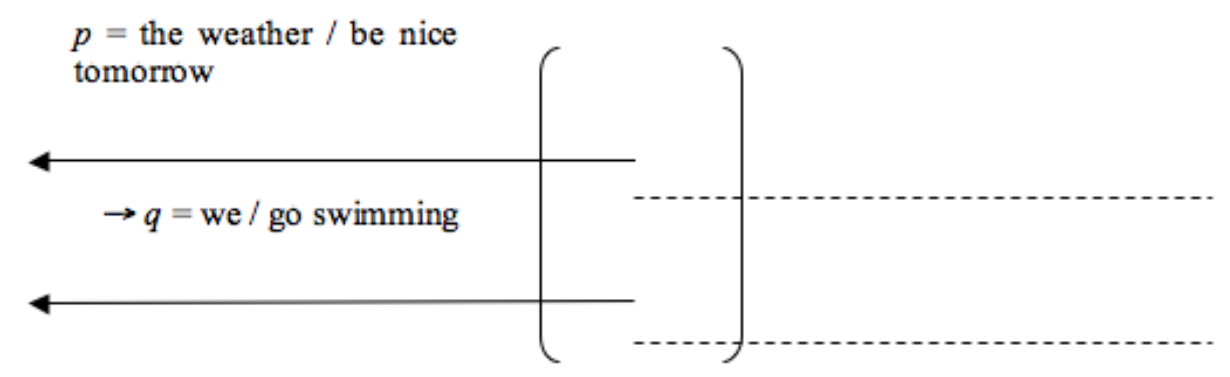

B. Application de la représentation du domaine notionnel au cas de l'exemple (1).

Or, pour bien cerner les phénomènes en jeu en ce qui concerne l'incompatibilité entre will et certaines subordonnées, il est également nécessaire de prendre en compte des critères spécifiques en fonction de la nature des subordonnées concernées. Ainsi, en ce qui concerne les relatives en when, nous avons proposé dans un précédent travail (Guillaume 2006) de considérer que l'opposition quantitative entre situation présente et situation à venir ( $1^{\text {er }}$ mouvement) se double d'une opposition au niveau qualitatif ( $2^{\text {ème }}$ mouvement) :

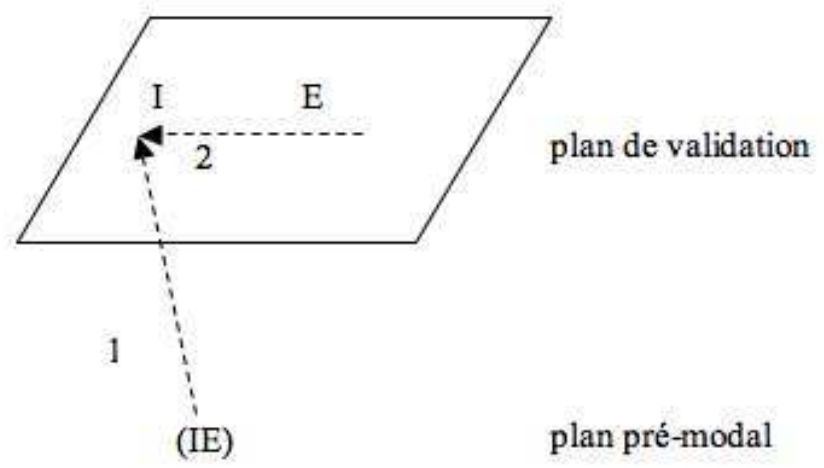

C. Représentation des opérations en jeu lors de l'emploi de will épistémique dans les subordonnées en when (cf. Guillaume 2006 : 73).

Le plan pré-modal contient la position décrochée (IE) (Culioli 1990 :162), position à partir de laquelle on peut envisager aussi bien la validation que la non-validation de la relation prédicative. Ceci correspond à la visée marquée par l'emploi du modal will ${ }^{18}$, qui est essentiellement quantitative dans la mesure où ce qui est en jeu est la réalisation ou la non-réalisation de l'événement visé. Quant au plan de validation, il correspond à la représentation du domaine notionnel que nous avons explicitée plus haut: l'Intérieur n'est pas sélectionné d'emblée, on part de l'Extérieur pour atteindre l'Intérieur. La problématique est ici qualitative: la situation présente de l'énonciateur constitue un préconstruit contraire auquel vient s'opposer le contenu de la subordonnée. Ainsi, dans l'exemple Both Dr Aris and the university look forward to the day when Aung San Suu Kyi will be free to come to Oxford to collect her honorary degree ( Le Dr Aris tout comme l'Université attendent avec impatience le jour où Aung San Suu Kyi sera libre de venir chercher son diplôme honoris causa à Oxford.»), l'énonciateur ne se contente pas d'utiliser une relative introduite par when pour déterminer l'antécédent the day, il oppose également de manière explicite la situation présente correspondant au moment 
d'énonciation, qui voit la dissidente birmane être assignée à résidence, et la situation à venir espérée, à savoir la libération d'Aung San Suu Kyi. plupart du temps du complémentaire de $p^{20}$, comme nous avons essayé de le montrer dans notre analyse du premier exemple, et ceci explique selon nous le fait que will serait agrammatical dans bien des cas. Tournons-nous néanmoins vers d'autres cas de figure dans lesquels on rencontre au contraire un emploi parfaitement acceptable de will dans la subordonnée.

\section{Un contexte restrictif (type only... if)}

(4) Clearly, it is only worthwhile for the band to request a royalty examination if the potential claim will exceed the professional fees incurred.

$\rightarrow$ De manière évidente, le fait de réclamer des droits d'auteur n'est valable pour un groupe que dans le cas où le gain espéré sera supérieur aux honoraires d'avocat que cela entraîne.

(5) Some people think devolution will only work if it will advantage business with reduced taxation.

$\rightarrow$ Certaines personnes pensent que la dévolution ne marchera que si elle donne l'avantage aux entreprises qui bénéficient d'une fiscalité réduite.

Dans ces exemples, $q$ est repéré par rapport à $p$, mais il est également repéré explicitement par rapport à p' grâce à la présence de l'adverbe only dans la principale. On peut gloser ainsi le repérage qui est en jeu entre les propositions ${ }^{21}$ :

\section{if $p$, then $q$}

and if $p^{\prime}$, then non-q

Contrairement à l'exemple if the weather is nice tomorrow, dans lequel ce qui se passerait en cas de temps mauvais ou mitigé n'était pas énoncé explicitement, il est clair ici que l'action décrite dans la principale aura lieu exclusivement dans le cas où la condition énoncée dans la subordonnée est remplie. 
27 A noter qu'un contexte restrictif peut suffire à justifier l'apparition de will dans la subordonnée, sans forcément que l'adverbe only ou un synonyme n'apparaisse de manière explicite dans le contexte immédiat :

(6) In those circumstances the Crown Prosecution Service do not seek to be heard in this particular case. In any future case the situation will be considered on the facts known at that time and if appropriate application will be made.

$\rightarrow$ Dans ces conditions le Service des poursuites de la Couronne ne demande pas à être entendu sur ce cas précis. A l'avenir, les affaires ne seront prises en considération qu'en fonction des faits déjà connus et si une demande en bonne et due forme a été faite.

Ceci est à rapprocher également du cas de l'exemple (2), dont le contexte, en plus de faire apparaître comme nous l'avons vu une opposition à la fois temporelle et qualitative entre situation présente et situation future, comporte un contexte restrictif : l'intérêt de se livrer au calcul évoqué dépend avant tout de l'année de naissance.

Remarquons par ailleurs que l'emploi du présent simple dans la conditionnelle de (4), (5) et (6) respectivement ne serait en rien agrammatical. Il faut donc bel et bien considérer l'emploi du modal comme une intrusion modalisante de l'énonciateur dans son discours, qui est rendue possible par un contexte non-neutre.

\section{Renforcement (type especially if)}

Un autre cas dans lequel un adverbe (ou une paraphrase contenant cet adverbe) peut mettre en évidence le repérage de $q$ par rapport à p' est celui de l'emploi de especially ${ }^{22}$ :

(7) Fit a handrail or build parapet wall beside long flights, especially if they will be used by children or the elderly.

$\rightarrow$ Installez une rampe ou bien un garde-fou le long des escaliers de hauteur importante, a fortiori s'ils doivent être utilisés par des enfants ou des personnes âgées.

(8) It is generally easier to alter margins before you have typed in a document, especially if you will be setting tabs.

$\rightarrow$ Il est en général plus simple de modifier les marges avant de taper votre document, d'autant plus si vous prévoyez d'avoir recours à des tabulations.

31 La présence de only explicitait une restriction qui découlait d'un lien exclusif entre $p$ et $q$ : en l'absence de $p$, pas de $q$. Ici, en fait, c'est l'inverse : la réalisation de $p$ vient renforcer $q$, mais même en l'absence de $p$ on aurait $q$. On peut le formuler ainsi :

if $p^{\prime}$, then $q$

if $p$, then $q^{+}$

\section{Préconstruit contraire et éventualité}

(9) For desk lighting, provision of an individual lamp can be most helpful for those who are visually handicapped, although the availability of power points in the classroom may cause problems. However, if an individual lamp will help a child to succeed in an integrated setting, every effort should be made to provide one.

$\rightarrow$ En ce qui concerne l'éclairage des bureaux, le fait de mettre à disposition une lampe individuelle peut se révéler très utile pour les déficients visuels, bien que le nombre limité de prises de courant au sein d'une classe puisse poser problème. Néanmoins, si une lampe individuelle doit aider un enfant à réussir son intégration dans un environnement adapté, tous les efforts doivent être mis en cuvre pour en fournir une. 
Contrairement à ce qui se passe avec les exemples concessifs que nous allons examiner par la suite, il n'y a ici aucune contradiction entre $p$ (if an individual lamp will help a child to succeed in an integrated setting) et $q$ (every effort should be made to provide one); on est confronté au contraire à un lien de cause à effet typique des conditionnelles. En revanche, il y a dans le contexte un préconstruit contraire venant s'opposer à $q$, à savoir the availability of power points in the classroom may cause problems. On peut formuler ainsi les opérations en jeu:

$$
\text { if } p^{\prime} \text {, then } q^{\prime} \rightarrow \text { if } p \text {, then } q
$$

On en revient donc à la relation toute simple donnée au départ (if $p$, then $q$ ), mais il s'agit là en fait d'une reformulation dans un contexte qui, au départ, laissait présager la mise en avant de la relation inverse. Un tel phénomène est comparable à l'opposition à l'œuvre dans le marquage des références temporelles étudiée précédemment (exemples 2 et 3 ) : il y a là encore un préconstruit contraire, mais celui-ci est dû à la nature des événements décrits et non à leur référence temporelle (voir ce qui se passe au niveau du plan de validation dans le schéma $\mathrm{C}$ ). A nouveau, ces exemples ne peuvent fonctionner qu'au sein d'un contexte explicite, et par rapport aux implications présentes dans ce dernier.

La modalisation effectuée par l'emploi de will souligne une évolution, qui se traduit par le passage mental d'une situation de départ vers une situation d'arrivée a priori en contradiction avec la première. Un tel mouvement ne serait pas traduit par l'emploi du présent simple: sans être agrammatical, le remplacement de will par le présent appauvrirait le sémantisme de la subordonnée. Dans ce type d'exemples, le préconstruit contraire en contexte peut appartenir à l'Extérieur ou bien à la Frontière, comme c'est le cas en (9):

\section{INTERIEUR}

FRONTIERE

\section{EXTERIEUR}

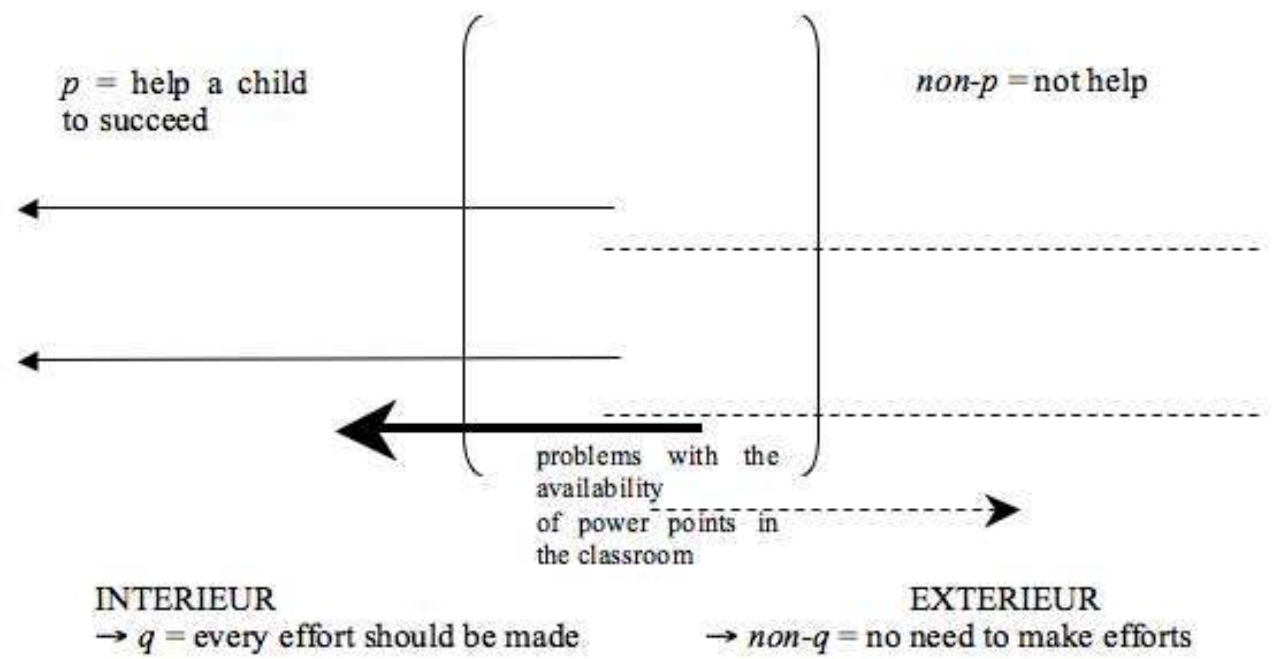

D. Application de la représentation du domaine notionnel au cas de l'exemple (9).

La flèche en gras permettant une translation de la Frontière vers l'Intérieur vient s'opposer à la flèche en pointillés, qui correspond pour sa part à ce que l'on pourrait déduire du préconstruit contextuel (matérialisé sur notre schéma dans la Frontière du domaine de q), et qui pour autant n'est pas sélectionnée au final ${ }^{23}$.

En revanche, dans l'exemple suivant, qui, comme nos exemples du BNC, est authentique, mais que nous empruntons cette fois-ci à R.A. Close (1980:104), un préconstruit contraire 
existe par rapport au contenu de la subordonnée et non plus de la principale. Par ailleurs, la Frontière du domaine notionnel correspondant à $p$ peut cette fois-ci être représentée par un trait, aucune occurrence du contexte ne venant a priori l'instancier ${ }^{24}$ :

(10) If you will be alone on Christmas Day, let us know now.

$\rightarrow$ Si toutefois vous risquez de vous retrouver seul le jour de Noël, faites-le nous savoir dès maintenant.

INTERIEUR

$p=$ be alone for Christmas
FRONTIERE

EXTERIEUR

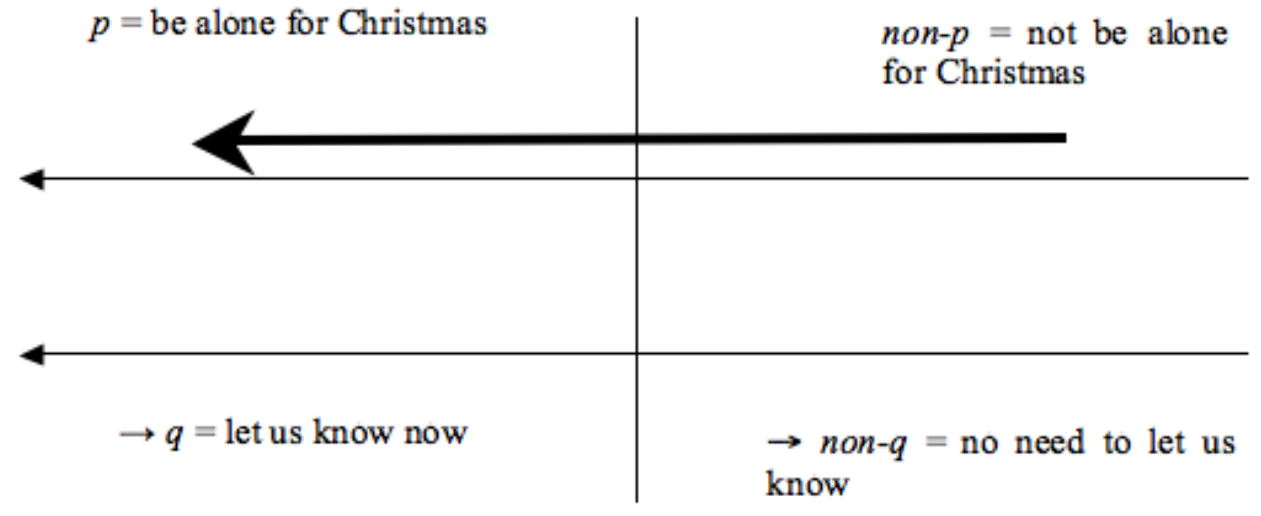

E. Application de la représentation du domaine notionnel au cas de l'exemple (10).

Le préconstruit contraire n'est pas verbalisé ici, car il est en réalité culturel : Christmas Day évoque famille et convivialité, il y a donc une réelle dissonance à l'associer à be alone. C'est donc non-p qui est présenté implicitement comme étant la valeur privilégiée. Néanmoins, en dépit de ce préconstruit, l'énonciateur choisit la valeur $p$ dans la subordonnée, ce qui a pour conséquence d'entraîner $q$ : let us know now.

Par ailleurs, les exemples que l'on peut placer dans cette catégorie contiennent une insistance particulière sur l'évaluation des chances de réalisation de la relation prédicative contenue dans la subordonnée $p$. Ceci rejoint les observations de Close sur la prévisibilité supposée (1980 : 107-9), dont il a déjà été question :

(11) If the death penalty will save a single life, it is justified.

$\rightarrow$ Si la peine de mort doit sauver ne serait-ce qu'une seule vie, elle se justifie.

(12) If you need to pause to think before answering, do so, and if you stumble over an answer, start again if that will help.

$\rightarrow$ Si vous avez besoin de faire une pause pour réfléchir avant de répondre, faites-le, et si vous butez sur une réponse, recommencez depuis le début si cela doit vous aider.

(13) I need your help very badly, Mr Holmes. If it will be useful for Dr Watson to hear what I have to say, please let him stay and listen.

$\rightarrow$ J'ai désespérément besoin de votre aide, M. Holmes. Si cela doit être utile au Dr Watson

d'écouter ce que j'ai à dire, je vous en prie, qu'il le fasse.

Ainsi, dans l'exemple (11), l'évaluation des chances de réalisation de $p$ est assez faible, sans toutefois être nulle, comme le montre le recours à une occurrence minimale : a single life. Or, même si les chances de voir l'événement décrit par la subordonnée se produire sont extrêmement faibles, au point que cela pourrait ne se passer qu'une seule fois, l'estimation est jugée suffisante par l'énonciateur pour entraîner la conséquence contenue dans la principale. Une fois de plus, l'évaluation qui est faite du contenu de la subordonnée, n'est pas donnée d'emblée : évaluée comme faible dans un premier temps (jugement quantitatif), elle est en fin de compte valorisée comme étant non-nulle 
(jugement qualitatif). Cette évolution dans l'appréciation est mieux rendue par le dynamisme de will ${ }^{25}$ que par le statisme du présent aoristique.

En revanche, dans les exemples (12) et (13), les chances de réalisation de $p$ ne sont pas évaluées au départ comme particulièrement faibles. Néanmoins, le mécanisme est le même : le fait que l'événement décrit dans $p$ ait la moindre chance de se réaliser justifie la mise en œuvre de $q$.

\section{Phénomène de reprise}

41 Avant de nous intéresser à certaines concessives, examinons un cas de conditionnelle qui, contrairement aux catégories passées en revue précédemment, ne dépend pas du repérage par rapport au complémentaire linguistique. Il y a néanmoins à nouveau une prise en compte importante des implications du contexte puisque le contenu de la subordonnée en if fait écho à un discours antérieur ${ }^{26}$, qui peut être réel ou fantasmé, et, à ce titre, l'emploi de will s'y trouve être plus mimétique que réellement significatif :

(14) If legislation is needed to get the treaty through Parliament, if it is so popular with the country, and if it will be such a wonderful thing for unemployed people, for women, for the regions and for business, why does not the Prime Minister dissolve Parliament next week and have a general election in January?

$\rightarrow$ S'il faut une loi pour faire ratifier ce traité par le Parlement, si le projet de traité est tellement apprécié des électeurs, et si cela doit être tellement merveilleux pour les chômeurs, pour les femmes, pour les régions et pour le commerce, pourquoi le Premier Ministre ne dissout-il pas le Parlement la semaine prochaine afin d'organiser de nouvelles élections législatives en janvier?

(15) President Mengistu Haile Mariam, in the course of a three-hour broadcast speech on April 19, offered to resign 'if that will preserve the unity of the country'.

$\rightarrow$ Le Président Mengistu Haile Mariam, lors de la retransmission d'un discours de trois heures le 19 avril, a proposé de démissionner "si cela doit sauvegarder l'unité du pays ».

L'exemple (14) est ironique, comme le montre l'exagération dans le choix du vocabulaire ( such a wonderful thing). L'énonciateur caricature l'enthousiasme du parti au pouvoir pour son propre projet; ce faisant, il fait mine de reprendre verbatim une expression qu'ils n'ont peut-être jamais employée en réalité. En revanche, dans l'exemple (15), le journaliste se contente de citer les propos du dictateur déchu, comme le montre l'emploi de guillemets.

Dans l'exemple suivant, c'est l'expression the most popular remark qui évoque une forme de polyphonie qui pourrait remplir le critère de la reprise d'un discours antérieur :

(16) We were very silent, at the Post, when the news of Stalingrad reached us. If only this will shorten things a bit, was the most popular remark.

$\rightarrow$ Nous demeurâmes très silencieux, au siège du Post, lorsque nous fûmes mis au courant des nouvelles de Stalingrad. Si seulement ça pouvait accélérer un peu les choses, entendaiton le plus souvent.

Cet énoncé comporte de surcroît l'expression if only ${ }^{27}$, une tournure qui pourrait apparaitre également assez facilement dans le contexte de (15). On pourrait presque parler ici d'un emploi optatif de la subordonnée en if ${ }^{28}$. Par ailleurs, en (16), l'emploi du modal s'oppose moins à celui du présent simple qu'à celui du prétérit: if only that shortened things a bit... Dans cette paraphrase néanmoins, l'éventualité est présentée comme plus aléatoire ; will met au contraire en avant la visée de l'événement, et donc sa prévisibilité, pour reprendre le terme de R.A. Close. 
45 A. Celle explique pour sa part que l'emploi de la modalité prend, dès lors qu'elle peut être attribuée à un autre énonciateur, un "caractère objectif» (2003: 79-80). En cela, elle reprend la distinction entre modalité épistémique objective et modalité épistémique subjective que propose de faire J. Lyons (1977: 805 ; voir aussi Chuquet 2001: 168-9 et Huddleston \& Pullum $2002: 191$, note 58). On n'est dès lors plus vraiment dans le cadre de l'emploi de will dans une conditionnelle tel qu'il a été analysé jusqu'à présent; il ne s'agit plus à proprement parler d'une modalisation, et on est finalement assez proche de l'emploi aoristique du présent. Une telle analyse ne s'applique néanmoins qu'à une toute petite minorité des exemples de notre corpus, notamment (14) et (15).

Par ailleurs, (14) présente également des points communs avec un phénomène déjà rencontré, dans les exemples (2) et (3) plus précisément. En effet, il est lui aussi le lieu d'un contraste purement temporel entre le moment d'énonciation et le moment ultérieur auquel la loi en question entrera en vigueur (on sous-entend : once implemented). Quant à (15) et (16), ils peuvent aussi être rapprochés de phénomènes analysés précédemment. On y note en effet la présence implicite d'un préconstruit contraire ( $c f$. les exemples 9 et 10), dans la mesure où l'énonciateur semble quelque peu douter de la réalisation de l'événement espéré ; à ce titre, ils présentent également une insistance particulière sur l'évaluation des chances que l'événement décrit dans la subordonnée puisse effectivement avoir lieu (cf. les exemples 11 à 13).

47 Nous n'avons en effet pour notre part trouvé aucun exemple dans lequel la présence de will dans la subordonnée se justifierait exclusivement par la reprise d'un discours antérieur: il nous semble donc qu'il s'agit là d'un phénomène facilitant l'emploi de ce modal, sans toutefois pouvoir le déclencher à lui tout seul.

Intéressons-nous maintenant à certaines concessives introduites par if. Dans un tel cas de figure, l'emploi de will est admis sans restriction, quelle que soit sa valeur (radicale ou épistémique). Il est néanmoins intéressant d'examiner le repérage inter-propositionnel qui se met alors en place, dans la mesure où il se révèle assez proche de ce que l'on a observé jusqu’à présent dans les conditionnelles.

\section{Subordonnées en if ayant une valeur concessive}

G. Ranger (1998 : 28-32) définit une relation de concession comme étant la déconstruction d'un lien inférentiel attendu entre $p$ et non- $q$, de manière à aboutir à une absence de relation privilégiée entre $p$ et non- $q$, ce qui correspond à la valeur de rupture (ou décrochage) de l'opérateur de repérage dans la $\mathrm{TOE}^{29}$. Prenons un exemple tiré de notre corpus :

(17) If the economy will not defeat the regime, it will not save it either.

$\rightarrow$ Si l'économie ne causera pas la perte du régime, elle ne le sauvera pas non plus.

Tout comme les conditionnelles, les concessives jouent sur la mise en relation de deux relations prédicatives. Il se révèle là encore pertinent de représenter le domaine notionnel de chacune de ces relations; on peut par ailleurs matérialiser par une flèche rouge le décalage qui s'effectue entre ce qui était prévisible au départ et ce qui est énoncé au final : 
INTERIEUR

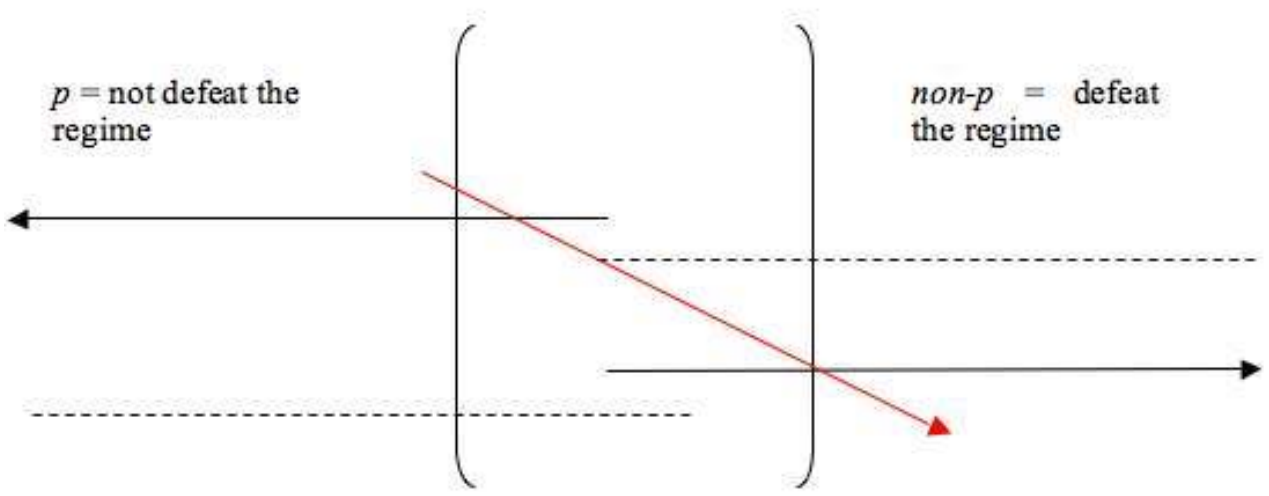

EXTERIEUR

$\rightarrow$ non $-q=$ save the regime
EXTERIEUR

$n o n-p=$ defeat

the regime

F. Application de la représentation du domaine notionnel au cas de l'exemple (17).

aurait pu logiquement déduire de la proposition $p$ : the economy will not defeat the regime une proposition non-q qui serait the economy will save the regime; or, en réalité, on passe de $p$ à $q$, c'est-à-dire: the economy will not save the regime. On peut proposer la formule suivante :

\section{if $p$, then $q \rightarrow$ if $p$, then non- $q$}

A nouveau, la présence d'une flèche sur notre schéma permet de montrer symboliquement que les opérations qui sous-tendent l'énoncé sont foncièrement dynamiques ${ }^{30}$ ( $c f$. schémas $\mathrm{C}$, D et E). Or, un modal tel que will, qui est fondamentalement un marqueur de visée, fonctionne forcément mieux dans de tels contextes que le présent aoristique $^{31}$, plus statique et moins plastique du point de vue des opérations en jeu.

Le même mécanisme est à l'œuvre en (18) : la critique du style de l'auteur contenue dans la subordonnée concessive en if n'implique pas pour autant que le style en question ne comporte que des défauts. On retrouve du reste un marqueur typique de la concession dans l'emploi de l'expression adverbiale at least :

(18) The truth may be that her stoical-witty-rueful literary persona may even steer the writer towards the sort of life that ensures her material doesn't dry up. If she will never be able to hide under the beauty of her image clusters, at least she will never bore us with the undergrowth of psychology and metaphor, where art is an excuse for waffle.

$\rightarrow$ La vérité est sans doute que son personnage littéraire, tout à la fois stoïque, plein d'esprit et piteux, peut même diriger l'écrivain vers le style de vie qui fait en sorte que la substance de son inspiration ne s'épuise pas. Si elle ne pourra jamais s'effacer derrière la beauté de ses métaphores filées, au moins elle ne nous ennuiera jamais avec des considérations psychologisantes et métaphoriques, où l'art n'est qu'une excuse pour produire du verbiage.

\section{Concessives introduites par even if ${ }^{32}$}

Dans une approche énonciative, le propre de even est de signaler une «valeur ultime avant le passage à E » (Culioli 1999b : 177-8). En réalité, cette valeur limite n'appartient pas à l'Intérieur strict $p$ mais à la Frontière, et donc au complémentaire linguistique $p$ '. La représentation topologique du domaine notionnel permet de visualiser ceci : 
(19) You have got off lightly, even if your face will be swollen and painful for some time ${ }^{33}$.

$\rightarrow$ Vous vous en êtes tiré à bon compte, même si votre visage va rester enflé et douloureux pendant quelque temps.

INTERIEUR

FRONTIERE

EXTERIEUR

get off lightly

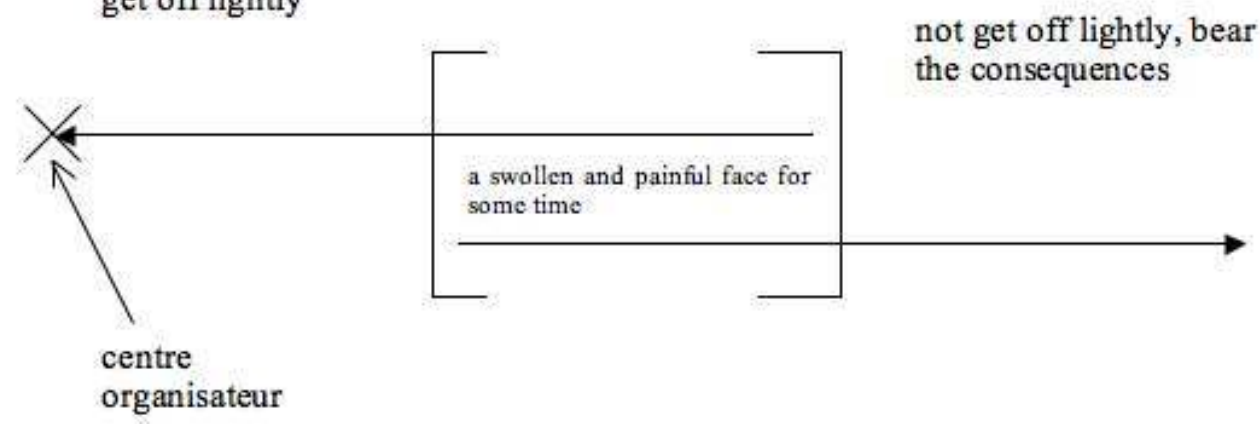

G. Application de la représentation du domaine notionnel au cas de l'exemple (19).

A. Culioli note que l'emploi de even devant if "déclenche une valeur d'inefficacité " (1999b : 179) dans la mesure où la subordonnée, qui ressemble superficiellement à une conditionnelle en if, n'a en réalité aucune prise sur la validation de la relation contenue dans la principale. On peut proposer la formule suivante en ce qui concerne le repérage entre les propositions :

\section{if $p$, then $q$}

even if $p^{\prime}$, then $q$

A noter qu'il s'agit-là en quelque sorte de l'image-miroir de la relation postulée en ce qui concerne la catégorie précédente, avec cette fois-ci $q$ qui reste stable tandis que l'on passe de $p$ à $p$.

\section{Conclusion}

57 L'examen de ces quelques concessives introduites par if nous permet de conclure ce rapide tour d'horizon. Il aurait toutefois été possible de faire le lien avec d'autres cas d'emploi de will dans une subordonnée introduite par if, telle les interrogatives indirectes ou bien les comparatives en as if. On pourrait en effet montrer que de tels cas de figure correspondent là encore à la prise en compte du complémentaire linguistique. Ainsi, en ce qui concerne les interrogatives, il s'agit là d'un cas bien connu de parcours entre les deux pôles (Intérieur et Extérieur) du domaine notionnel ${ }^{34}$. Quant au fonctionnement de as if, il permet de produire un "ajustement énonciatif entre deux réalités en conflit " (Culioli 1999b : 180-1), et à cet égard il se révèle proche du mécanisme de la concession. 


\section{BIBLIOGRAPHIE}

\section{Principales références:}

BOUSCAREN, Janine et CHUQUET, Jean. 1987. Grammaire et textes anglais. Guide pour l'analyse linguistique. Paris : Ophrys.

CELLE, Agnès. 2003. «La visée dans les propositions hypothétiques en anglais et en français. » La Subordination en anglais. Une approche énonciative. Eds A. Celle et S. Gresset : 69-87. Toulouse : Presses Universitaires du Mirail.

CHUQUET, Jean. 1984. «If... ». Cahiers de Recherche en grammaire anglaise. Tome 2. Gap : Ophrys. 45-87.

---. 2001. « Modalité et Subordination. » Modalité et opérations énonciatives. Cahiers de Recherche tome 8. Eds J. Bouscaren, A. Deschamps et L. Dufaye : 3-22. Paris : Ophrys.

CLOSE, R.A. 1980. "Will in if-clauses." Studies in English Linguistics for Randolph Quirk. Eds S. Greenbaum, G. Leech et J. Svartvik : 100-9. Londres : Longman.

COMRIE, Bernard. 1985. Tense. Cambridge Tetxbooks in Linguistics. Cambridge : Cambridge UP. COTTE, Pierre. 1988. Le Système des auxiliaires modaux dans le système verbal de l'anglais contemporain. Thèse de Doctorat d'Etat. Université de Grenoble III.

CULIOLI, Antoine. 1990. Pour une linguistique de l'énonciation. Opérations et représentations. Tome I. Paris : Ophrys.

---. 1999a. Pour une linguistique de l'énonciation. Formalisation et opérations de repérage. Tome II. Paris : Ophrys.

---. 1999b. Pour une linguistique de l'énonciation. Domaine notionnel. Tome III. Paris : Ophrys.

DECLERCK, Renaat. 1984. “"Pure future' will in if-clauses.” Lingua $63: 279-312$.

DE MATTIA-VIVIES, Monique. 2000. Le discours indirect en anglais contemporain. Approche énonciative. Aix en Provence : Publications de l'Université de Provence.

---. 2006. Le discours indirect libre au risque de la grammaire : Le cas de l'anglais. Aix-enProvence : Publications de l'Université de Provence.

DESCHAMPS, Alain. 1999. «Essai de formalisation du système modal de l'anglais. » Les opérations de détermination. Quantification / Qualification. Eds A. Deschamps et J. Guillemin-Flescher : 269-85. Paris : Ophrys.

---. 2001. « Approche énonciative des modaux de l'anglais. » Modalité et opérations énonciatives. Cahiers de Recherche tome 8. Eds J. Bouscaren, A. Deschamps et L. Dufaye : 3-22. Paris : Ophrys.

DE VOGÜE, Sarah. 1987. « Si, la condition nécessaire et la condition suffisante. » Actes du colloque sur l'implication dans les langues naturelles et dans les langages artificiels Université de Strasbourg 1985. Paris : Klincksieck. 
---. 1999. « Le champ des subordonnées dites conditionnelles du français : conditions, éventualités, suppositions et hypothèses. » L'Hypothétique, LINX n 41 : 93-118. Paris : Université Paris X Nanterre.

DUBOIS, Jean et al. 1999. Dictionnaire de linguistique et des sciences du langage. Nouvelle édition. Paris : Larousse-Bordas.

DUFAYE, Lionel. 2002. «La représentation de l'irréel : de l'intuition aux opérations. » Anglophonia / Sigma. 12 : 29-61. Toulouse : Presses Universitaires du Mirail.

GILBERT, Eric. 1993. « La théorie des opérations énonciatives d'Antoine Culioli. » Les théories de la grammaire anglaise en France. Ed. P. Cotte : 63-96. Paris : Hachette Supérieur.

---. [1998] 2001. « A propos de WILL. », Les Verbes modaux. Eds P. Dendale et J. Van Der Auwera. Cahiers Chronos $8: 123-39$. Amsterdam : Rodopi.

---. 2001a. « Vers une analyse unitaire des modalités. » Modalité et opérations énonciatives. Cahiers de Recherche tome 8. Eds J. Bouscaren, A. Deschamps et L. Dufaye : 23-100. Paris : Ophrys. GROUSSIER, Marie-Line et RIVIERE, Claude. 1996. Les mots de la linguistique. Lexique de linguistique énonciative. Paris : Ophrys.

GUILLAUME, Bénédicte. 2006. «Will dans les subordonnées en when est-il un marqueur de différenciation au niveau qualitatif ? » Cycnos. 23.1 : 63-75.

---. 2011. « Application du concept de Frontière à l'emploi de la modalité de visée dans certaines subordonnées en anglais. » Cahiers de Praxématique 53 : La notion de Frontière notionnelle et de son utilité pour l'analyse linguistique. Coord. S. Mellet. Montpellier : PU de l'Université PaulValéry - Montpellier III.

HUDDLESTON, Rodney et PULLUM, Geoffrey. 2002. The Cambridge Grammar of the English Language. Londres : Cambridge UP.

LARREYA, Paul. 1991. Le possible et le nécessaire : Modalités et auxiliaires modaux en anglais britannique. Paris : Nathan Université.

LEECH, Geoffrey. 1971. Meaning and the English Verb. Londres : Longman.

LYONS, John. 1977. Semantics 2. Cambridge : Cambridge UP.

MOREAU, Catherine. 2003. «L'hypothèse de l'irréel ». Journée sur l'irréel. Université Paris IV.

PALMER, Frank Robert. 1979. Modality and the English Modals. Londres : Longman.

RANGER, Graham. 1998. Les constructions concessives en anglais : Une approche énonciative. Numéro spécial des Cahiers de Recherche. Paris : Ophrys.

---. 2007. « Le concept de frontière : applications en linguistique énonciative. » L'identité et ses frontières : approches croisées d'un malaise contemporain. Ed. M. Beauviche. Avignon : Université d'Avignon et des Pays de Vaucluse.

---. 2008. « All this if not more... » L'envers du décor. Etudes de linguistique anglaise. Ed. Geneviève Girard-Gillet : 239-254. Avignon : Editions Universitaires d'Avignon.

ROUSKOV-LOW, Jennifer. 2001. “A Prosodic Approach to Modality.” Modalité et opérations énonciatives. Cahiers de Recherche tome 8. Eds J. Bouscaren, A. Deschamps et L. Dufaye : 255-82. Paris : Ophrys.

WYLD, Henry. 2001. Subordination et énonciation. Numéro spécial des Cahiers de Recherche. Gap : Ophrys. 
Corpus :

British National Corpus. 2000. World edition. The Humanities Computing Unit of Oxford University.

\section{NOTES}

1. Nous exprimons notre reconnaissance aux membres de l'équipe « énonciation » de l'UMR BCL, équipe dirigée par Sylvie Mellet, ainsi qu'aux participants à l'atelier «linguistique " (ALAES) présidé par Geneviève Girard-Gillet lors du XLIXème congrès de la SAES (Université de Bordeaux III, 8-10 mai 2009) pour les remarques, critiques constructives et suggestions de références formulées suite à la présentation de versions antérieures de cet article. Nous restons néanmoins seule responsable de toute erreur ou inexactitude.

2. Les exemples considérés ici concernent uniquement la forme présente de ce modal; voir Guillaume 2011 pour l'étude d'exemples de conditionnelles introduites par if et comportant would.

3. Par exemple: Angelo offers her a bargain: if she will sleep with him her brother shall live (BNC; "Angelo lui propose un marché : si elle accepte de coucher avec lui, son frère sera épargné. »). Nous considérons en effet que, dans de tels exemples, will est sélectionné en raison de son sémantisme (comme le montre du reste la traduction en français, en général par «accepter » pour will, et par « refuser » pour will + négation), et non en tant que modalisateur. On sort donc de la problématique qui nous intéresse ici.

4. Ce corpus d'anglais britannique comporte cent millions de mots et a été réuni par les Universités d'Oxford et Lancaster, en collaboration avec la British Library et des maisons d'édition. Nous utilisons la World Edition de l'an 2000.

5. Il s'agit-là de l'acronyme de SGML Aware Retrieval Application.

6. A savoir la manifestation d'un positionnement marqué de l'énonciateur vis-à-vis de son propos : « la modalisation définit la marque donnée par le sujet à son énoncé, c'est la composante du procès d'énonciation permettant d'estimer le degré d'adhésion du locuteur à son énoncé » (Dubois et al. 1999 : 305).

7. On rencontre également l'appellation «subordonnée hypothétique». Or, Sarah de Vogüé souligne l'« élasticité » de ces désignations en fonction des auteurs, et explique que « la catégorie est parfois rassemblée au titre de la condition, parfois au titre de l'hypothèse " tout en «[associant] généralement les deux» (1999: 95). D'autres auteurs (par exemple Dufaye 2002, Moreau 2003...) parlent pour leur part de l'expression de l'irréel. Nous n'entrerons pas dans le débat, qui ne rejaillit pas directement sur notre propos ici, et faisons dans l'ensemble un emploi peu sophistiqué du terme " conditionnelle».

8. Ceci est d'autant plus vrai en l'absence de tout contexte, celui-ci se révélant déterminant dans la possibilité d'employer will, comme nous allons essayer de le montrer.

9. Au contraire, dans le cas des subordonnées temporelles, le futur simple ne pose pas de problème en français, tandis que l'emploi de will est pour sa part soumis à des restrictions en anglais (cf. Guillaume 2006).

10. Sauf mention contraire, tous les exemples cités par la suite sont également extraits du BNC. Les traductions proposées sont les nôtres.

11. Il ne faut bien sûr pas tenir compte de la date de 1998, dans la mesure où le texte date d'une époque antérieure à cette année-là.

12. Nous remercions Paul Larreya de nous avoir indiqué cette référence.

13. Sur l'aoristique, voir Culioli 1999a : 127-43.

14. En revanche, nous ne partageons pas la conclusion à laquelle ils arrivent concernant le caractère « objectif » de la modalité épistémique marquée par will dans de tels exemples. Il nous 
semble au contraire que l'évaluation des chances qu'a un événement de se réaliser est une opération mentale particulièrement subjective, à moins de la comprendre dans le cadre d'un phénomène de reprise - dès lors, la subjectivité est celle de l'énonciateur rapporté et non celle de celui qui formule la conditionnelle. Voir à ce propos le commentaire des exemples (14) et (15).

15. Voir par exemple la définition donnée par Dubois et al. 1999 : 388.

16. Voir notamment Culioli 1990 : 67-71 ; 83-90 ; 95-99.

17. «Dans la représentation topologique de la notion comme domaine notionnel de Culioli, l'Intérieur strict $\mathrm{p}$ (en tant qu'ouvert, c'est-à-dire sans la Frontière) a, en principe, pour complémentaire l'Extérieur non-p + la Frontière (c'est-à-dire l'Extérieur en tant que fermé). On désigne alors le complémentaire par le symbole p'. » (Groussier et Rivière 1996: 41; c'est nous qui mettons en gras).

18. Pour plus de détails à ce sujet, voir Bouscaren et Chuquet $1987: 52$, ainsi que les schémas proposés par A. Deschamps (2001: 6-7), dans lesquels le modal will est symbolisé par une bifurcation pointant vers l'Intérieur, tant sur les plans quantitatif que qualitatif.

19. J. Rouskov-Low (2001) explique en effet que le choix de la forme pleine et / ou accentuée des modaux de l'anglais plutôt que de la forme réduite implique une plus grande complexité des opérations énonciatives en jeu, ce qui correspond à la modalisation de l'ensemble de l'énoncé et au contexte non-neutre que nous évoquons pour la plupart des exemples étudiés dans cet article. 20. A. Culioli explique ainsi à propos de l'assertion fictive en général : « Avec if, on construit l'existence fictive de $\mathrm{p}$, en relation avec un second terme, $\mathrm{q}$, conséquent au premier. On aboutit ainsi à une relation d'entraînement ( $\mathrm{p}$ entraîne $\mathrm{q}$ : si $\mathrm{p}$, alors $\mathrm{q}$ ), où l'on ne dit rien de p' (complémentaire de p)» (1999b : 179 ; c'est nous qui mettons en gras).

21. Si de telles notations sont utilisées en logique formelle, il s'agit simplement dans le cadre du présent travail d'expliciter le repérage entre la principale et la subordonnée et non de rendre compte de manière rigoureuse des implications logiques et / ou pragmatiques.

22. En ce qui concerne l'ordre des propositions, on constate que l'antéposition de la subordonnée impliquerait la suppression de especially, ce qui semble suggérer dans ce cas de figure un ordre contraint, tant au niveau syntaxique qu'au niveau opérationnel.

23. Voir à titre de comparaison le schéma que propose G. Ranger afin de représenter l'opération associée à l'emploi du marqueur concessif aun / aùn en espagnol (2007 : 13).

24. Selon les cas en effet, la Frontière peut être vide ou bien épaisse.

25. Par le «dynamisme » de will, nous entendons la possibilité qu'offre ce modal non seulement de viser la bonne valeur I sur le plan quantitatif, sans pour autant éliminer totalement $\mathrm{E}$, en raison du décalage temporel entre moment d'énonciation et moment de référence, mais également, sur le plan qualitatif, d'atteindre $p$ en partant de $p$ ', ce qui est particulièrement visible dans nos exemples. Voir schéma $\mathrm{C}$ et note 17.

26. Sur les problématiques proprement linguistiques liées à l'analyse des divers types de discours rapporté, voir M. De Mattia-Viviès 2000 et 2006.

27. A ne pas confondre bien sûr avec le cas de only... if abordé précédemment.

28. F.R. Palmer donne l'exemple inventé suivant : If only people will vote in sufficient numbers to put the Liberals back! dans lequel will exprime selon lui la volition (1979:117). Il ne peut s'agir de cela en (16), puisque le sujet en est inanimé ; néanmoins, la valeur radicale, de type propriété du sujet, prend de manière évidente largement le pas sur la valeur épistémique dans cet exemple (16), qui est donc à la marge de notre propre étude.

29. A propos de la valeur de rupture, voir Groussier et Rivière 1996 : 71-2.

30. Voir note 25.

31. L'emploi du présent simple dans la subordonnée de l'exemple (17) ne serait pas agrammatical mais changerait le sens: il s'agirait alors d'envisager une possibilité et d'en tirer une conséquence, autrement dit on se retrouverait dans la cadre d'une conditionnelle classique plutôt que d'une concessive. 
32. Pour l'analyse d'autres exemples de l'emploi de will dans une subordonnée introduite par even if, voir Guillaume 2011.

33. Contrairement à ce qui se passait pour les exemples avec especially, on peut ici envisager d'intervertir les deux propositions, ce qui aurait seulement pour effet de donner plus d'importance à la subordonnée qu'elle n'en a dans l'énoncé d'origine.

34. Voir notamment Culioli $1990: 132$ à propos du lien entre l'interrogation, le parcours sur tout le domaine, et l'emploi de «si » en français.

\section{RÉSUMÉS}

A partir d'un corpus d'une centaine d'exemples en anglais tirés du British National Corpus, dans lesquels on rencontre l'emploi du modal will au sein d'une subordonnée introduite par if, nous nous intéressons au fonctionnement de ce modal dans des subordonnées conditionnelles (par exemple, it will work only if it will advantage business ou encore if the death penalty will save a single life ...), et également dans quelques concessives (if the economy will not defeat the regime, it will not save it either). En revanche, nous ne nous intéressons pas au cas dans lequel will est utilisé principalement pour exprimer la volition (if she will sleep with him...).

Nous souhaitons montrer que l'emploi de will dans de tels exemples permet avant tout à l'énonciateur d'effectuer une modalisation de son énoncé, qui ne traduit pas tant un jugement subjectif de sa part que la reformulation d'implications présentes au sein d'un contexte foncièrement non-neutre. Ces implications sont parfois liées à la problématique du marquage des références temporelles (if you will be away on holiday), sans pour autant que cela soit toujours le cas, loin s'en faut. En réalité, le point commun est que, dans la plupart des cas, la principale $q$ se trouve être repérée non seulement par rapport à $p$, autrement dit la subordonnée introduite par if (en référence à la formule if $p$, then $q$ ), mais également par rapport à $p$, c'est-à-dire le complémentaire linguistique de la relation prédicative contenue dans la subordonnée. Par ailleurs, les composantes de la tentative de typologie que nous proposons ici ne sont pas hermétiques les unes par rapport aux autres, dans la mesure où plusieurs facteurs peuvent se combiner afin d'expliquer le recours à will dans la subordonnée. Le cadre théorique auquel il est fait référence est celui de la Théorie des Opérations Enonciatives d'Antoine Culioli.

This paper is based upon a corpus of about one hundred examples, drawn from the British National Corpus, of the use of the modal auxiliary will in some conditional if-clauses (for instance, it will work only if it will advantage business or if the death penalty will save a single life...), and also in a few concessive ones (if the economy will not defeat the regime, it will not save it either). The case in which will is used primarily to express volition (if she will sleep with him...) is not taken into account.

I try to demonstrate that the use of the modal in the examples under consideration is meant to express the speaker's attitude to the propositional content of the utterance as a whole, which is not so much the speaker's personal opinion as the translation of the sometimes conflicting implications of a non-neutral context. From a more technical point of view, it can be shown that most of the time $q$, the main clause, is not located only with respect to $p$, the subordinate ifclause (as exemplified in the well-known formula: if $p$, then $q$ ), but also to $p$, its linguistic complement. Time reference can also be an important factor in some of the examples studied (if you will be away on holiday), but they remain, in fact, a minority. I also put forward a typology of 
the main cases involved, bearing in mind that the components of such a typology must overlap one another since it often occurs that more than one phenomenon can account for the use of the modal in the subordinate. As far as the theoretical background is concerned, extensive reference is made to the linguistic concepts of A. Culioli's utterer-centred approach.

INDEX

Keywords : if- subordinates, will, tenses, modality, conditional clauses, concessive clauses, enunciation

Mots-clés : subordonnées en if, will, temps, modalité, subordonnées conditionnelles, subordonnées concessives, énonciation

\section{AUTEUR}

\section{BÉNÉDICTE GUILLAUME}

Université de Nice - Sophia Antipolis et UMR CNRS 6039 Bases, Corpus et Langage (BCL) 